\title{
Growth of defect-free colloidal hard-sphere crystals using colloidal epitaxy
}

Tonnishtha Dasgupta, John R. Edison, and Marjolein Dijkstra

Citation: The Journal of Chemical Physics 146, 074903 (2017); doi: 10.1063/1.4976307

View online: http://dx.doi.org/10.1063/1.4976307

View Table of Contents: http://aip.scitation.org/toc/jcp/146/7

Published by the American Institute of Physics

\section{Articles you may be interested in}

Island nucleation and growth with anomalous diffusion in one-dimension

The Journal of Chemical Physics 146, 074702074702 (2017); 10.1063/1.4976137

Reconciling transition path time and rate measurements in reactions with large entropic barriers

The Journal of Chemical Physics 146, 071101071101 (2017); 10.1063/1.4977177

Does the configurational entropy of polydisperse particles exist?

The Journal of Chemical Physics 146, 014502014502 (2017); 10.1063/1.4972525

Colloidal transport by active filaments

The Journal of Chemical Physics 146, 024901024901 (2017); 10.1063/1.4972010

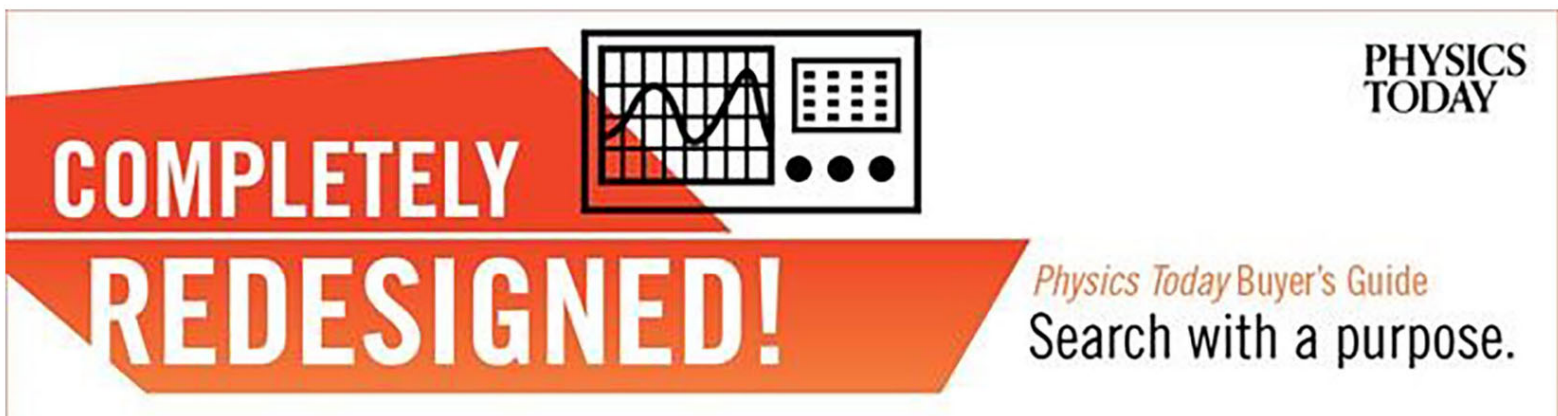




\title{
Growth of defect-free colloidal hard-sphere crystals using colloidal epitaxy
}

\author{
Tonnishtha Dasgupta, ${ }^{1}$ John R. Edison, ${ }^{2, a)}$ and Marjolein Dijkstra ${ }^{2, b)}$ \\ ${ }^{1}$ Soft Condensed Matter, Debye Institute for Nanomaterials Science, Department of Physics, Utrecht University, \\ Princetonplein 5, 3584 CC Utrecht, The Netherlands \\ ${ }^{2}$ Soft Condensed Matter, Utrecht University, Princetonplein 5, 3584 CC, Utrecht, The Netherlands
}

(Received 4 October 2016; accepted 23 January 2017; published online 21 February 2017)

\begin{abstract}
Using event-driven Brownian dynamics simulations, we investigate the epitaxial growth of hardsphere crystals with a face-centered-cubic (fcc) structure on the three densest cross-sectional planes of the fcc: (i) fcc (100), (ii) fcc (111), and (iii) fcc (110). We observe that for high settling velocities, large fcc crystals with very few extended defects grow on the fcc (100) template. Our results show good agreement with the experiments of Jensen et al. [Soft Matter 9, 320 (2013)], who observed such large fcc crystals upon centrifugation on an fcc (100) template. We also compare the quality of the fcc crystal formed on the fcc (111) and fcc (110) templates with that of the fcc (100) template and conclude that the latter yields the best crystal. We also briefly discuss the dynamical behavior of stacking faults that occur in the sediments. Published by AIP Publishing. [http://dx.doi.org/10.1063/1.4976307]
\end{abstract}

\section{INTRODUCTION}

Template assisted sedimentation, more commonly known as colloidal epitaxy, is a simple and robust approach to grow single crystals of colloidal particles, a key component of next generation optoelectronic devices. ${ }^{1}$ The ability to study the structure and dynamics of such systems in real space using confocal microscopy lends valuable information to understand the growth and defect dynamics at the atomic scale in the growth of atomic semiconductor materials. ${ }^{2-4}$ For the above stated reasons, sedimentation of colloidal hard-sphere fluids has been extensively investigated by both experiments and simulations. As is well known, the hard-sphere model captures several equilibrium and non-equilibrium phenomena of condensed matter systems. Computer simulations established that the stable crystal phase of hard spheres exhibits the facecentered cubic (fcc) structure. ${ }^{5-8}$ However, this structure is only slightly more stable than the hexagonal close-packed (hcp) crystal structure with a free-energy difference on the order of $10^{-3} k_{B} T$ per particle at the melting transition point $\left(k_{B}\right.$ is Boltzmann's constant and $T$ is the temperature). This small free-energy difference leads to the formation of the randomhexagonal-close-packed (rhcp) structure instead of a perfect single fcc crystal. Even though an rhcp structure restructures into the fcc crystal over long periods of time, ${ }^{9-11}$ methods to rapidly grow large colloidal crystals are highly desirable. Fabricating large defect-free single crystals, which are technologically significant, has posed severe challenges even in the simple case of hard spheres. A complete understanding of the sedimentation behavior of hard sphere-like systems would further provide clues on how to selectively grow a chosen crystal structure from a set of competing polymorphs with similar values of free-energy.

\footnotetext{
a) Present address: Molecular Foundry, Lawrence Berkeley National Laboratory, 1 Cyclotron Road, Berkeley, CA 94720, USA.

b) Electronic mail: m.dijkstra@uu.nl
}

Here we briefly discuss a few experimental studies on the sedimentation of such colloidal hard spheres on flat walls. Experimental realizations of the hard-sphere model are achieved by index matching a suspension of colloidal particles. ${ }^{12,13}$ The key factors that affect the quality of the crystalline sediment are (i) the initial volume fraction $\eta_{i}$ of the suspension and (ii) Peclet number $\mathrm{Pe}=m g \sigma / k_{B} T$, where $g$ is the gravitational acceleration, $\sigma$ the particle diameter, and $m$ the buoyant mass of the particles. Typically in experiments, $\eta_{i}$ is kept low to ensure that crystal growth is nucleated and directed by the wall. Hoogenboom et al. experimentally studied the sedimentation of hard-sphere-like silica colloids on a flat wall. ${ }^{14,15}$ For sufficiently low $\eta_{i},{ }^{14}$ they observed the formation of a predominantly fcc crystal. They showed that the sedimentation flux given by $J=\eta_{i} \mathrm{Pe}$ is the key variable that determines the fraction of fcc stacked layers in the sediment. In the regime of very low particle flux, the crystallizing layers have sufficient time to equilibrate and the fraction of fcc stacked layers is large, whereas predominantly randomly stacked hexagonal layers are found if $J$ is high. ${ }^{7,14,16,17}$ In density matched hard-sphere suspensions or under microgravity conditions, ${ }^{18,19}$ typically the rhcp structure is observed. Therefore the predominant presence of fcc domains in the slowly grown samples of the sediment is surprising. As noted earlier, the difference in free energies between the fcc and hcp structures is very small; however, fcc is the stable structure. Pronk and Frenkel $^{9}$ calculated the fcc-hcp interfacial freeenergy and used it to estimate the time that it takes to form a pure fcc crystal from the randomly stacked phase. They estimated that the healing of stacking faults in micrometer sized hard-sphere crystallites occurs on the time scale of days. The slow growth rates together with the slightly low free-energy of the fcc structure might have tipped the balance towards fcc in the experiments of Hoogenboom et al. ${ }^{14}$ It is also possible that in the presence of a gravity field, the fcc structure is more stable than in bulk, or that the dynamic pathways of crystallization under gravity could lead predominantly to the formation 
of fcc domains. Another explanation for this observation was provided by Hillhorst et al. ${ }^{20}$ Using confocal microscopy, they showed that these fcc domains often contained slanted stacking faults, which are two subsequent hcp stacked layers

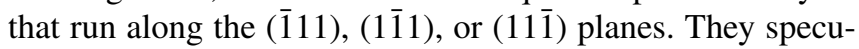
lated that these faults direct the formation of the fcc crystal domains.

Using computer simulations, Marechal et al. ${ }^{21}$ studied the crystallization dynamics of hard spheres sedimenting on a flat wall. Note that in these simulations and in our work, the role of the solvent is taken into account through the Brownian noise, and hydrodynamic effects are not treated rigorously. Nevertheless, their simulations showed that slow sedimentation favors the growth of fcc crystals over rhcp crystals, for low initial volume fractions, similar to the experimental findings of Hoogenboom et al. ${ }^{14}$ Furthermore, they showed that the quality of the formed fcc crystal depends on the combined effect of initial volume fraction and Peclet number just as shown by Hoogenboom et al. A good quality fcc crystal is a single crystal which is not divided into different crystallite domains and contains very few defects. They attributed the formation of predominantly fcc-like domains to the free-energy difference between fcc and hcp and not to the slanted stacking faults found in the fcc regions.

The introduction of patterned templates in place of a flat wall, together with a slow deposition rate, has been shown to direct layer by layer growth of the crystal with very few defects. $^{22-27}$ The template can often drastically reduce the metastability of the crystallizing fluid. ${ }^{28}$ One of the first noteworthy studies on colloidal epitaxy is that of Van Blaaderen et al. ${ }^{22}$ They demonstrated experimentally that under slow sedimentation, templates can be used effectively to grow large defect free crystals and control the lattice spacing and orientation of the resulting crystals. They also found that sedimenting hard spheres on an isotropically deformed hcp (1100) template resulted in the epitaxial growth of a perfect hcp crystal. $^{25,26}$

In the case of flat walls, slow sedimentation rates are preferred to avoid amorphization of the sediment, ${ }^{29}$ but how the scenario changes when the crystal is grown epitaxially remained unexplored until recently. Jensen $e t$ al. looked at the sedimentation of hard-sphere-like fluids for a wide range of sedimentation velocities on flat walls, fcc (100), fcc (111), and the fcc (110) template. ${ }^{27}$ They showed that large defect-free fcc colloidal crystals can be grown by centrifuging at surprisingly high speeds of up to $3000 \mathrm{~g}$ onto a (100) oriented fcc (square) template. These findings are particularly attractive as they describe a way to rapidly grow fcc crystals with only a few extended defects, as large time scales of slow sedimentation are undesirable. Their findings also raise interesting questions on the growth mechanism and the dynamics of defects, if any, that occur in crystals grown at very large sedimentation velocities. They observed that this was unique to the fcc (100) template and noted that the absence of stacking degeneracy might be the cause.

Motivated by these experiments, we present in this paper a computer simulation study of the crystallization dynamics of hard-sphere fluids under an applied gravitational field in the presence of three different crystalline templates. Using a series of order parameters as described by Marechal et al. ${ }^{21}$ we characterize the sediment and identify optimal conditions for the growth of large defect-free fcc crystals. This paper is organized as follows. In Section II, we describe the simulation methods, including the Event-Driven Brownian Dynamics (EDBD) simulations and the order parameters that we use. We present our results in Section III. We first discuss our simulation results for an fcc (100) template, which gives by far the best quality fcc crystals even at high sedimentation velocities. We then discuss sedimentation on different templates and end with some conclusions in Section IV.

\section{SIMULATION METHODS}

\section{A. Systems studied}

We use EDBD simulations ${ }^{30,31}$ in the $N V T$ ensemble to simulate a system of $N$ hard spheres with a diameter $\sigma$ in a volume $V$ subject to a gravitational field. The method consists of computing a sequence of collision events that involves only two particles at any instant. During the simulation, stochastic adjustments of the particle velocities are periodically made at an interval of $\Delta t$ according to

$$
\mathbf{v}_{f}(t=s \Delta t)=\alpha_{t} \mathbf{v}_{0}(t)+\beta_{t} \mathbf{v}_{R}(t),
$$

where $\mathbf{v}_{0}(t)\left(\mathbf{v}_{f}(t)\right)$ is the velocity of the particle before (after) the stochastic adjustment or thermalization, $s$ is any integer, $\mathbf{v}_{R}(t)$ a $3 \mathrm{D}$ Gaussian variable with mean 0 and variance $k_{B} T / m$ with $m$ the mass of the particle, $k_{B}$ the Boltzmann constant, and $T$ the temperature. We set $\alpha_{t}=1 / \sqrt{2}$ with a probability $v \Delta t$ and 1 otherwise. We set $\beta_{t}=\sqrt{1-\alpha_{t}^{2}}$ in order to keep the temperature constant. Following Ref. 21, we set $v=10 \tau_{M D}^{-1}$ and $\Delta t=0.01 \tau_{M D}$ in all our simulations, where $\tau_{M D}=\sqrt{m / k_{B} T} \sigma$ is the time unit of an event-driven MD simulation. The details of the EDBD simulations are elaborately described in Refs. 21 and 31 .

The spheres are confined between a template (or a smooth wall) at $z=0$ and another smooth wall at $z=H$. The height $H$ of the column is chosen such that the density of the colloids at the top of the sedimentation column is almost negligible when the sedimentation-diffusion equilibrium is reached. The template particles are not fixed but carry a reduced weight 1000 times that of the sedimenting particles. Therefore, their positions remain unaltered and the structure of the template remains fixed during the course of the simulation. We perform simulations for a system size of $N=100000$ particles with the cross-sectional area of the simulation box fixed at $\simeq 50 \sigma \times 50 \sigma$. The simulation box is periodic in the $x$ and $y$ directions. The gravitational field is oriented along the $z$-direction, and the colloids experience an external potential $\phi(z)=m g z$ with $g$ the gravitational acceleration and $z$ the vertical coordinate of the colloid. The parameter that describes the effect of the gravity field on the particles is the "gravitational length," given as $l_{g}=k_{B} T / m g$. It is a measure of the length scale over which the effect of gravity manifests. ${ }^{32}$ Another dimensionless parameter of interest is the gravitational Peclet number, $\mathrm{Pe}=m g \sigma / k_{B} T$. As we keep $\sigma$ fixed in our simulations, a change in the value of Pe changes the gravitational length. 
In this work, we simulate sedimentation on three different templates for $\mathrm{Pe}=0.5,1,2,3,4,5$, and 10. The higher the Peclet number, the faster is the sedimentation and the shorter the time available for the particles to rearrange and equilibrate. As already mentioned in the Introduction, another relevant parameter for sedimentation is the initial volume fraction $\eta_{i}$ of the suspension. We perform simulations with an initial volume fraction $\eta_{i}=0.01,0.02$, and 0.104 . The figures that we present below correspond to simulations using an initial volume fraction $\eta_{i}=0.02$, unless otherwise stated. The initial configurations of our simulations are homogeneous fluid phases of non-overlapping hard spheres which fill the entire sedimentation column.

\section{B. Order parameters}

In this section we briefly describe the order parameters, as described by Marechal et al., ${ }^{21}$ that we use to identify the differently oriented fcc and hcp structures in the sediment. The fcc and hcp crystal structures differ from each other by the stacking sequence of the hexagonal layers formed by the particles. To determine if a particle is solid-like, we estimate its local symmetry by using bond orientational order parameters. The un-normalized 3D bond order parameter for particle $i$ is defined as

$$
q_{l, m}^{(u)}(i)=\frac{1}{N_{n b}(i)} \sum_{j=1}^{N_{n b}(i)} Y_{l, m}\left(\theta_{i, j}, \phi_{i, j}\right),
$$

where $N_{n b}(i)$ denotes the number of neighbors of particle $i$, $\theta_{i, j}$ and $\phi_{i, j}$ are the polar and azimuthal angles, respectively, of the center-of-mass distance vector $\mathbf{r}_{i j}=\mathbf{r}_{j}-\mathbf{r}_{i}$ with $\mathbf{r}_{i}$ the position of particle $i . Y_{l, m}(\theta, \phi)$ are the spherical harmonics for $m$ ranging from $[-l, l]$. The summation runs over all neighboring particles $j$, which we define as the particles that lie within a center-of-mass distance of $1.3 \sigma$ with respect to $i$. The normalized bond order parameter is defined as

$$
q_{l, m}(i)=\frac{q_{l, m}^{(u)}(i)}{\left(\sum_{m=-l}^{l}\left|q_{l, m}^{(u)}(i)\right|^{2}\right)^{1 / 2}} .
$$

A neighbor $j$ of particle $i$ forms a solid-like bond with $i$ if $d_{l}(i, j)>d_{c}$ with

$$
d_{l}(i, j)=\sum_{m=-l}^{l} q_{l, m}(i) q_{l, m}^{*}(j) .
$$

Solid-like or crystalline particles are defined as particles for which the number of solid-like bonds $n_{\text {con }}(i)$ is greater than a critical value $n_{c} \cdot{ }^{33}$ The symmetry index in the above equations is chosen as $l=6$. Following Marechal et al. ${ }^{21}$ we set the cut-off values defined above as $n_{c}=4$ and $d_{c}=0.7$.

Upon identifying solid-like particles, we then identify its crystal structure. The fcc (111) and hep crystals are composed of stacked hexagonal layers. Therefore, we use two-dimensional bond order parameters to quantify the hexagonal symmetry of the layers. For a particle $i$, this is given by

$$
\psi_{m}^{S}(i)=\frac{1}{N_{s}(i)} \sum_{j=1}^{N_{s}(i)} \exp \left(m i \varphi_{i j}\right),
$$

where the sum over $j$ runs over the $N_{s}(i)$ neighbors in environment $S$ of particle $i$ and $\varphi_{i j}$ denotes the angle between the projection of $\mathbf{r}_{i j}$ on the $x-y$ plane and the $x$-axis. The $x-y$ plane is defined as the plane parallel to the template (perpendicular to gravity). Because of the in-plane hexagonal order of these two crystals, we chose the symmetry index to be $m=6$.

The difference between the fcc (111) and hcp crystal structures is the stacking sequence of hexagonal layers. While fcc (111) shows ABC $\cdots A B C$ stacking of layers, the stacking sequence in a hcp structure is $\mathrm{ABA} \cdots \mathrm{BAB}$. Thus, after confirming the hexagonal symmetry of the layers, the next step is to calculate a "stacking parameter" $\alpha$ that distinguishes the two different kinds of stacking. To determine the stacking parameter $\alpha_{i}$ for a given particle $i$, we look at three different environments of a particle $i$.

The environment $S=1$ corresponds to the neighbors $j$ in the layer above particle $i$ as defined by $0.55 \sigma<\left(z_{j}-z_{i}\right)$ $<1.2 \sigma$ and $r_{i j}=\left|\mathbf{r}_{i j}\right| \leq 0.95 \sigma$. The environment $S=-1$ comprises particles that are the neighbors $j$ in the layer below particle $i$ for which $-1.2 \sigma<\left(z_{j}-z_{i}\right)<-0.55 \sigma$ and $r_{i j}$ $\leq 0.95 \sigma$, and for environment $S=0$ the neighbors lie in the same layer defined by $\left|z_{j}-z_{i}\right|<0.5 \sigma$ and $r_{i j} \leq 1.3 \sigma$. The environment of a solid-like particle $i$ is hexagonally ordered in the same layer if $\left|\psi_{6}^{0}(i)\right|>0.7$. The stacking can only be defined if the layers above and below particle $i$ are also hexagonally ordered and are a part of the same crystalline domain, i.e., $\psi_{6}^{\text {surr }}(i) \equiv\left|\psi_{6}^{1}(i)+\psi_{6}^{-1}(i)\right|>0.5$.

Once the hexagonal order of the domain is ascertained, the stacking can be determined using the trigonal bond order parameter, which for a perfect fcc is $\psi_{3}^{f c c}(i)=\frac{1}{2} \mid \psi_{3}^{1}(i)$ $-\psi_{3}^{-1}(i) \mid=1$, while for a perfect hcp, $\psi_{3}^{h c p}(i)=\frac{1}{2} \mid \psi_{3}^{1}(i)$ $+\psi_{3}^{-1}(i) \mid=1$. The stacking parameter $\alpha$ is then defined as the fraction of fcc stacked particles, i.e., $\alpha=\psi_{3}^{f c c} /\left(\psi_{3}^{f c c}+\psi_{3}^{h c p}\right)$. Note that when the averaged value of $\alpha$ over all hexagonally ordered particles is 0.5 , it signifies perfectly random packing.

The other crystal structure that we characterize in this paper is the cubic fcc (100). In this case the layers that are stacked parallel to the $x-y$ plane exhibit square symmetry. Therefore, to characterize the order within each layer in the $z$-direction, we compute the two dimensional bond order parameter $\psi_{4}^{0}(i)$ given by Equation (5) with the symmetry index $m=4$.

We list the bond order parameters that we used and the respective threshold values to distinguish the various crystal structures in Table I. Particles which are crystalline in nature and are not identified as fcc (100), fcc (111), and hcp

TABLE I. Bond order parameters and corresponding threshold values for identifying fcc and hcp structures.

\begin{tabular}{lc}
\hline \hline Type of structure & Conditions on particle $i$ \\
\hline Crystalline & $n_{\text {con }}(i)>4$ \\
fcc $(100)$ & $n_{\text {con }}(i)>4, \psi_{6}^{0}(i)<0.7, \psi_{4}(i)>0.5$ \\
fcc $(111)$ & $n_{\text {con }}(i)>4, \psi_{6}^{0}(i)>0.7, \psi_{6}^{\text {surr }}(i)>0.5, \alpha(i)>0.5$ \\
hcp & $n_{\text {con }}(i)>4, \psi_{6}^{0}(i)>0.7, \psi_{6}^{\text {surr }}(i)>0.5, \alpha(i)<0.5$ \\
\hline \hline
\end{tabular}


crystal structures also appear in the sediment as discussed in Section III. These are observed to either belong to a stacking fault or some other crystal structure which has not been analyzed in this study. Such particles are termed as defects in Secs. III and IV and colored in lilac. In addition, to assess the quality of a crystalline sediment, we estimate the fraction of fcc particles $\phi_{f c c}=N_{f c c} / N_{c r}$ with $N_{f c c}$ being the number of particles that are fcc-like and $N_{c r}$ being the number of particles that are identified as solid-like.

\section{RESULTS AND DISCUSSION}

As mentioned in the Introduction, we study the epitaxial growth of the hard-sphere fcc crystal on three different templates. The (100), (111), and (110) cross-sectional planes of the fcc structure are the obvious choices for simple patterning because they represent the three densest crosssectional planes of the fcc crystal. The hexagonal template in Fig. 1(a) is the densest possible packing of particles on the template and is the (111) cross section of the fcc crystal. There are two ways in which the next layer of spheres can sit on this pattern (labelled as B and C in Fig. 1(a)), both of which are energetically degenerate. Therefore, the stacking sequence of the hexagonal layers is likely to be random. The (110) fcc template shown in Fig. 1(c) does not show this stacking degeneracy but is the least dense of all three templates. Being the closest packed template which does not show stacking degeneracy, the square (100) fcc template in Fig. 1(b) is therefore the most promising template for growing large fcc crystals.

\section{A. Sedimentation on the fcc (100) template}

Therefore, we first present the results on the sedimentation of hard spheres on the (100) plane of a close-packed fcc crystal, for varying Peclet numbers (Pe) and initial volume fraction $\left(\eta_{i}\right)$. First, we show visualizations of the final configurations of the sediment for (i) $\eta_{i}=0.02, \mathrm{Pe}=2$ (Fig. 2(a)) and (ii) $\eta_{i}=0.02, \mathrm{Pe}=10$ (Fig. 2(d)). The time taken for the sediment to crystallize and equilibrate is inversely related to the Peclet number. The snapshots of the final configurations presented in Figs. 2, 6, and 8 are taken at a time which is well after $\phi_{f c c}$ has equilibrated with respect to simulation time. The color of a particle in Fig. 2 indicates if it belongs to an fcc or a hcp crystalline domain, based on the criteria listed in Section II. Lilac-colored particles in the snapshots indicate defects and we observe from the snapshots (Figs. 2(a) and 2(d)) that the crystalline sediment is less defective for higher settling speeds, i.e., higher Pe. This observation, which agrees very well with experiments, ${ }^{27}$ is surprising because at high Pe there is less time available for the particles to equilibrate. At the growth front, the particles of the layer that crystallizes appear to precisely sit on the holes (possible lattice positions) left by the previous layer of particles even under rapid sedimentation.

Next, we take a closer look at the dynamics of the sedimentation process. In Figs. 2(b), 2(c), 2(e), and 2(f), we show the time evolution of each layer of the sediment for both $\mathrm{Pe}=2$ and $\mathrm{Pe}=10$. The horizontal axis denotes the time scale $t / \tau_{M D}$ and the vertical axis indicates a layer $l$ of the sediment. We color the plots according to the values of $\phi_{f c c}^{l}$ or $\phi_{d e f}^{l}$, which we define as follows. $\phi_{f c c}^{l}\left(l, t / \tau_{M D}\right)$ is the fraction of similarly oriented

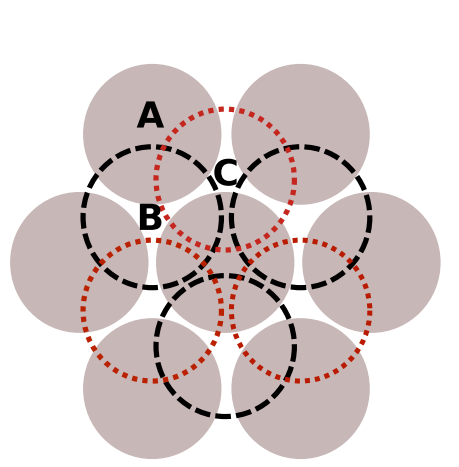

(a)

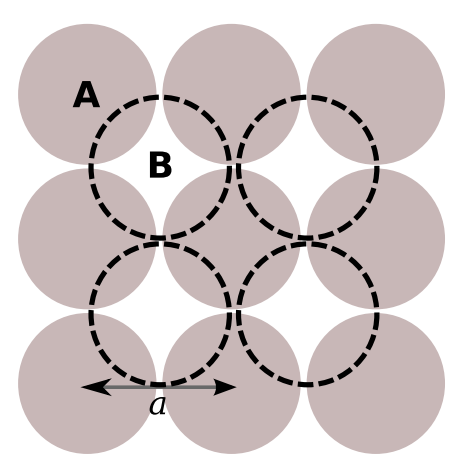

(b)

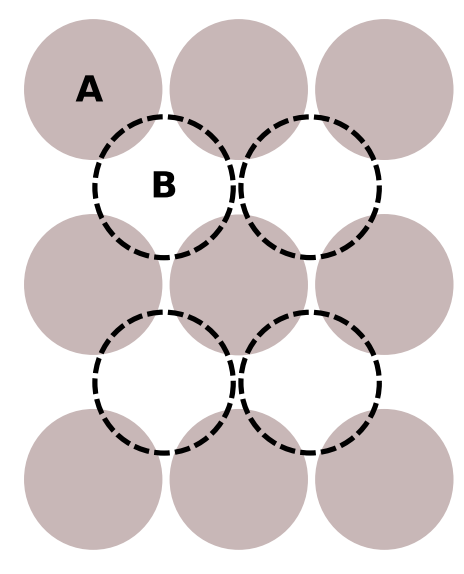

FIG. 1. Stacking degeneracy on different fcc templates. (a) Hexagonal fcc (111) template, (b) square fcc (100) template, (c) fcc (110) template. "A" (drawn as filled circles) represents the first layer of particles. "B" and "C," shown as black and red dashed hollow circles, respectively, in (a), are the two degenerate stacking positions for the second layer of particles. For (b) and (c), "B" shown as black dashed circles represents the second layer of particles on top of layer A. $a$ is the lattice spacing of the square template. 


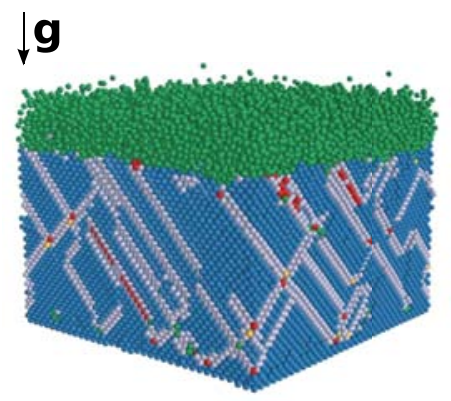

(a) $\mathrm{Pe}=2$

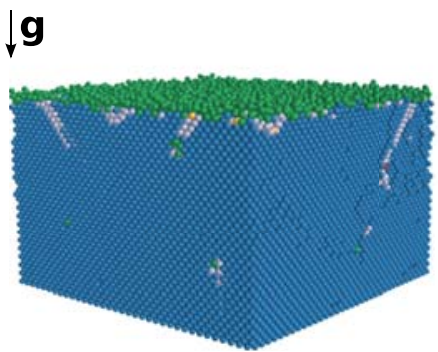

(d) $\mathrm{Pe}=10$

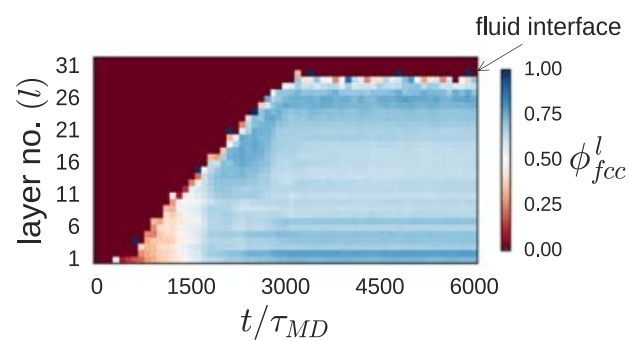

(b) $\mathrm{Pe}=2, \phi_{f c c}^{l}$

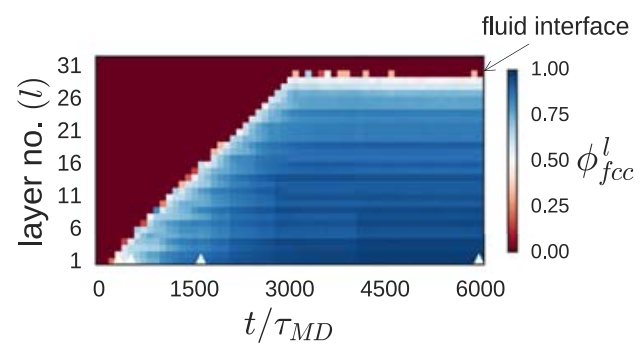

(e) $\mathrm{Pe}=10, \phi_{f c c}^{l}$

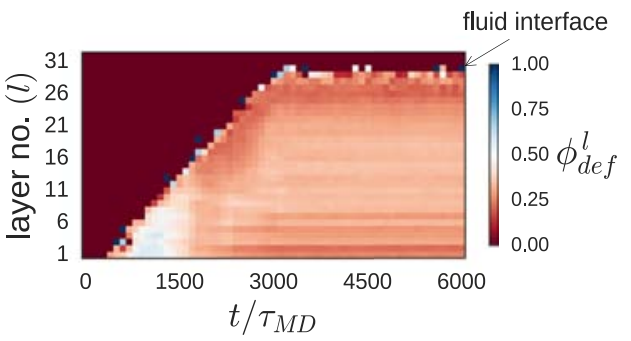

(c) $\mathrm{Pe}=2, \phi_{\text {def }}^{l}$

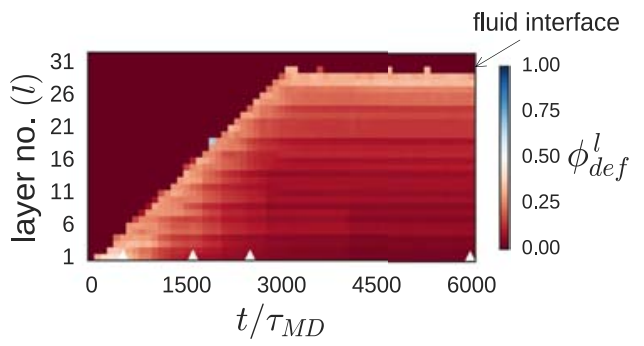

(f) $\mathrm{Pe}=10, \phi_{\text {def }}^{l}$

FIG. 2. ((a) and (d)) Side view of the final configurations at $t / \tau_{M D}=6000$ of hard spheres sedimenting on an fcc (100) template with lattice spacing $a=1.004 \sigma$ for Pe $=2$ and 10, respectively. The spheres in the snapshots are colored as follows: red: fcc (111), yellow: hcp, blue: fcc (100), lilac stacking fault, or another crystal structure, green: disordered. ((b) and (e)) Fraction of fcc particles $\phi_{f c c}^{l}$ as denoted by the color bar, calculated over the total number of crystalline particles in each layer, showing the crystallization of each layer with time. ((c) and (f)) Fraction of particles belonging to a fault/defect $\phi_{\text {def }}^{l}$ as denoted by the color bar, calculated over the total number of crystalline particles in each layer, plotted for each layer over time. The dark red region to the left of the four plots represents the absence of crystalline particles in the layer, i.e., a fluid layer. The initial packing fraction $\eta_{i}=0.02$.

fcc particles over the total number of crystalline particles in each layer of the sediment. Similarly, $\phi_{\text {def }}^{l}\left(l, t / \tau_{M D}\right)$ denotes the number of solid-like particles that are neither strictly fccnor hcp-like divided by the total number of crystalline particles in each layer. One can read the evolution of the crystallinity of a layer in time from such a plot by following the horizontal time axis for that layer. Similarly, one may also examine the crystalline nature of the entire sediment at a fixed instant of time by following the positive vertical axis from the bottom of the sediment to the top.

Based on Figs. 2(b) and 2(e), we make the following observations. The dark red regions to the top left of the plots correspond to disordered or fluid-like particles in the sediment. We clearly observe that the dark red region at the top shrinks with time as progressively more layers crystallize in the sediment. A deep blue region indicates a high fraction of fcc particles. Clearly, the crystal grown at $\mathrm{Pe}=10$ has a much higher fcc composition than the one for $\mathrm{Pe}=2$. The fraction of fcc in each layer increases as we go down the sediment for $\mathrm{Pe}=10$.

Moreover from Figs. 2(c) and 2(f), we observe that the sediment for $\mathrm{Pe}=10$ has far fewer defects than the one for $\mathrm{Pe}=2$. The nearly uniform light red color in Fig. 2(c) across the layers for the $\mathrm{Pe}=2$ case indicates the presence of a number of extended defects across layers, which do not anneal out in the simulation times studied. In contrast in Fig. 2(f), the deepening red color (indicating a decrease in $\left.\phi_{d e f}^{l}\right)$ from the top to the bottom layer for $\mathrm{Pe}=10$ represents few extended defects and progressively fewer stacking faults as we go down the sediment. If we follow the evolution of each layer in time, we can observe that there are further rearrangements of defects in the layers to form similarly oriented fcc particles.

For the Pe $=10$ case, from the very beginning, the layers crystallize rapidly into fcc particles, with the result that in a short amount of time a high fraction of fcc particles is formed. The crystalline sediment shows a linear growth front in time indicating that crystallization proceeds in a layerwise manner. As described earlier by Heni and Löwen, ${ }^{34}$ the mechanism behind the layer-by-layer growth of a crystal on a patterned template $e^{15,17,35-37}$ originates from the fact that the crystallised $(n-1)$ th layer serves as a template for the $n$th layer leading to its subsequent crystallisation. As the settling particles crystallize in a layer, some particles are stacked a little differently, at slightly higher positions than their neighbors in that layer. This may lead to a local stacking fault.

Next, we discuss the birth of these local stacking faults in the crystallizing layers and how they behave over time. In Figs. 3(a)-3(d), we present different snapshots in time of the first layer that is formed on top of the template for Peclet number $\mathrm{Pe}=10$. In the initial stages of crystallization, we observe numerous stacking faults as colored in lilac in Fig. 3. The stacking faults are formed when a crystalline domain, in which the particles are four-fold hollow-site stacked, is next to a domain where the particles are bridge-site stacked on the template. A 


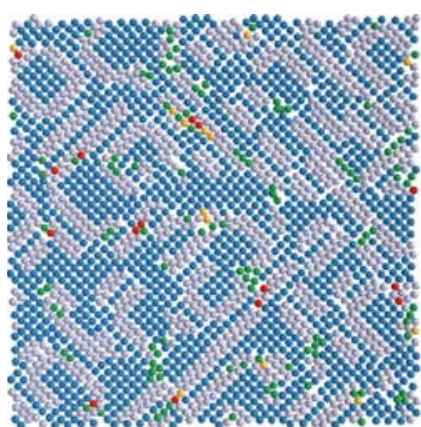

(a) $t / \tau_{M D}=300$

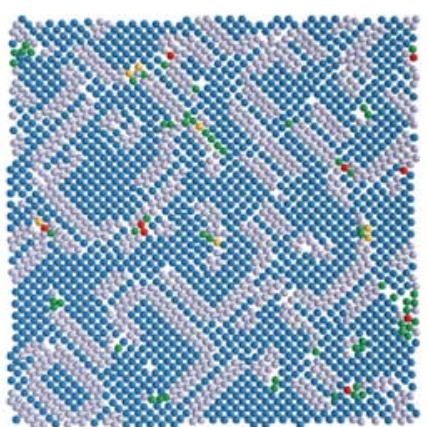

(b) $t / \tau_{M D}=500$

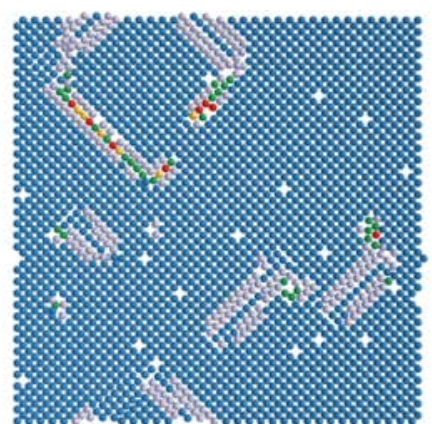

(c) $t / \tau_{M D}=1600$

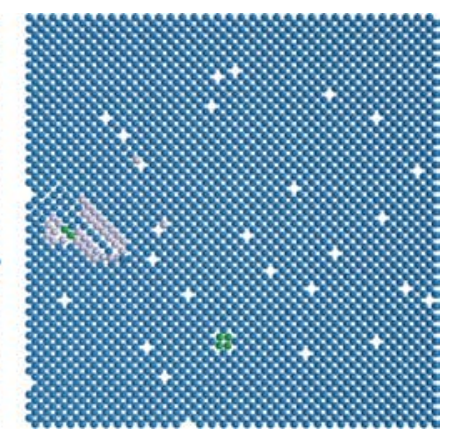

(d) $t / \tau_{M D}=6000$

FIG. 3. Typical configurations of the top view of the first layer of the crystalline sediment for different times as labeled for hard spheres sedimenting on a close-packed fcc (100) template at $\mathrm{Pe}=10$. The color code is the same as in Fig. 2.

hollow-site refers to a hole left by the particles of a layer and is therefore a lattice site for a particle in the next layer to sit. For a square template, the hollow-sites would have four-fold symmetry. A hollow-site stacked particle is therefore a perfectly stacked particle with regards to forming a perfect fcc crystal. A bridge-site is located anywhere on the line connecting the two in-plane particles (lattice sites) of a layer. Therefore, bridge-site stacked particles have to be slightly out of plane with respect to the particles in a layer but not exactly in the layer above. The bridge-site stacked particles are therefore at a higher height than the hollow-site stacked particles as shown in Figs. 4(a) and 4(b). In the course of sedimentation, the bridge-site stacked particles will be pushed towards the hollow-site positions by the weight of the particles that settle upon these particles, in order to minimize the gravitational potential energy. Here, it is important to note that in the case of flat walls, typically slow sedimentation rates are preferred to grow good crystals because there is more equilibration time available for particles to diffuse in-plane and therefore for defects to rearrange. ${ }^{29}$ However, for the close-packed fcc (100) template at high $\mathrm{Pe}(=10)$, there is negligible time for in-plane diffusion of particles and the annealing of defects in the layer is mainly governed by the gravitational pressure acting on the particles from the layers above. In this way the stacking faults anneal out fairly quickly, thereby leaving behind point vacancies in the crystalline layers. These point vacancies appear to be very persistent as they are in-plane with the layer and hard to anneal out when the next crystalline layer is already formed. It is worth noting that the confocal images of the crystalline layers in the colloidal crystals grown by centrifugation onto a (100) template at $3000 \mathrm{~g}$ also show numerous point vacancies, which is remarkably consistent with our observations

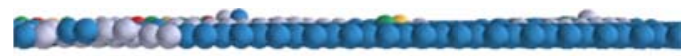

(a) $t / \tau_{M D}=1600$, side view, cropped

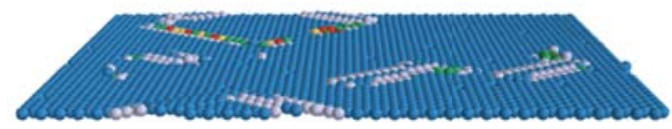

(b) $t / \tau_{M D}=1600$, tilted side view

FIG. 4. Side view (a) and tilted side view (b) of layer 1 at $t / \tau_{M D}=1600$ to show more clearly the stacking faults. The color code is the same as in Fig. 2. here. ${ }^{27}$ The simulation time that corresponds to the images in Figs. 3(a)-3(d) is marked in Figs. 2(e) and 2(f) by white triangles. We also looked at the evolution of layers 4 and 10 (not shown here) in our sediment and the behavior of the stacking faults is qualitatively similar to the dynamics of those in the first layer. As expected, there are more faults in the tenth layer which is higher up in the sediment because the lower the pressure these defects feel from the layers above the more difficult it is for these defects to rearrange. To illustrate this, we plot in Fig. 5 the fraction of crystalline defects $\phi_{\text {def }}^{l}$ in a layer $l$ as a function of the gravitational pressure $\left(\beta P \sigma^{3}(l)\right)$ on that layer for a well-equilibrated sediment at $t / \tau_{M D}$ $=6000$. We determine $\beta P \sigma^{3}(l)$ by integrating the density profile of the hard spheres along the sedimentation column. ${ }^{38}$ We clearly observe that $\phi_{d e f}^{l}$ decreases monotonically with the pressure.

Our results for the fcc (100) template show very good agreement with Jensen $e t a l .{ }^{27}$ who obtained single fcc crystals up to the top of the sediment by applying fields between $15 g$ and $3000 g$ via centrifugation. These single crystals are not broken up into smaller crystallites and contain few extended defects. Our simulation results also show that for the fcc (100) template, the higher the Peclet number, i.e., the faster the particles settle, the less defective the crystal (to compare Figs. 2(a) and 2(d)). This is surprising as it contrasts the results of sedimentation on a flat wall, where predominantly fcc-stacked crystals are observed only at sufficiently

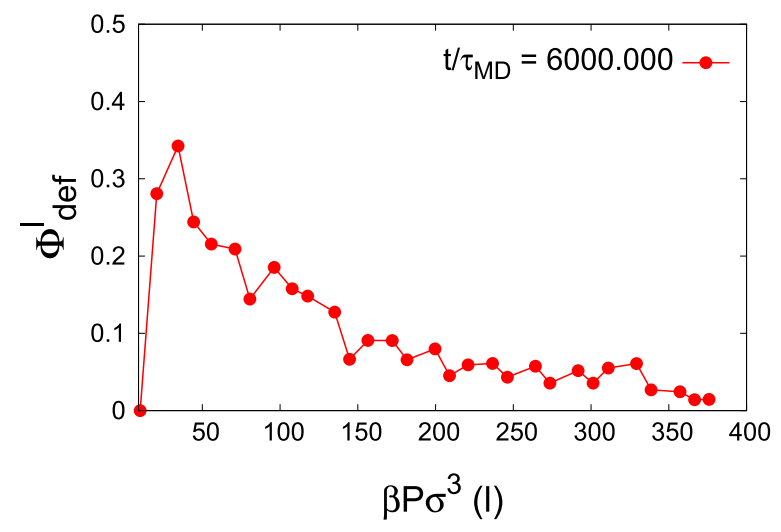

FIG. 5. The fraction of crystalline defects in each layer $\phi_{d e f}^{l}$ as a function of the gravitational pressure at that layer for a well-equilibrated sediment at $t / \tau_{M D}=6000$ on a $1.004 \sigma$ fcc $(100)$ template and $\mathrm{Pe}=10$. 
slow sedimentation rates ${ }^{14,21}$ and where disorder sets in at high Pe.

\section{B. Comparison with the flat wall, fcc (111), and fcc (110) templates}

Next we investigate the structure of the crystalline sediment using different cross-sectional planes of the closepacked fcc crystal as a template, i.e., the (i) fcc (111), (ii) fcc (110) crystal planes, as well as a (iii) flat wall for comparison.

The hexagonal fcc (111) template is the densest plane of the fcc crystal and one can expect that this might help direct the settling particles to the right lattice positions. However, for this template, there are two lattice sites on which the next layer of particles can sit, as shown in Fig. 1(a), giving rise to stacking degeneracy. We focus first on slow sedimentation rates, or the near-equilibrium growth regime $(\mathrm{Pe}=1)$, where the crystallizing particles get sufficient time to rearrange within the crystallizing layer to form a hexagonal layer with little or no defects. As mentioned earlier in the Introduction, stacking degeneracy for this template must result in a random stacking of hexagonal layers (rhcp) and this is what we observe. In Fig. 6(a) we show a snapshot of the sediment in the long time limit. Our observation is similar to experimental studies where it was observed that under microgravity conditions, the rhcp structure is formed. ${ }^{18}$

A more quantitative description of the dynamics is shown in Figs. 6(b) and 6(c). Note that in these graphs, the color bars represent the overall fraction of fcc $\left(\phi_{f c c}^{l}\right)$ and hcp $\left(\phi_{h c p}^{l}\right)$ particles in each layer, respectively. The colors in the two figures are complementary, i.e., for the deep blue layer in Fig. 6(b) we see a corresponding deep red layer in Fig. 6(c) and viceversa, showing that each layer is either fcc- or hcp-stacked. We also observe in certain layers that a smaller crystalline domain merges into and aligns itself with the largest growing planar $2 \mathrm{D}$ domain. This is shown in Figure 7. The deepening of color in time for certain layers in Figs. 6(b) and 6(c) is indicative of this process. Eventually this results in a fully hep- or fccstacked layer. From Fig. 6(b), it is evident that the quality of the fcc crystal is quite poor.

As discussed earlier, fcc and hcp crystals have a freeenergy difference of the order of $10^{-3} k_{B} T$ per particle in favor of fcc. In addition to the free-energy argument, Pronk et al. calculated the interfacial free-energy between fcc and hcp to be very low $\simeq 26 \pm 6 \cdot 10^{-5} k_{B} T / \sigma^{2}$, which favors the formation of a single fcc crystal. ${ }^{9}$ Therefore, we can expect the randomly stacked crystal to evolve into the fcc crystal over long periods of time. ${ }^{10,11}$ We do not see this transformation within the simulation times that we studied and expect it to occur at much longer time scales in the presence of a gravitational field than in bulk.

Next we present results on crystal growth at higher sedimentation speeds, i.e., Pe $=10$ in Figs. 6(d)-6(f). Looking at the snapshot, it is clear that the number of slanted stacking faults observed is very high. A slanted stacking fault is formed when two differently stacked domains grow on a horizontal

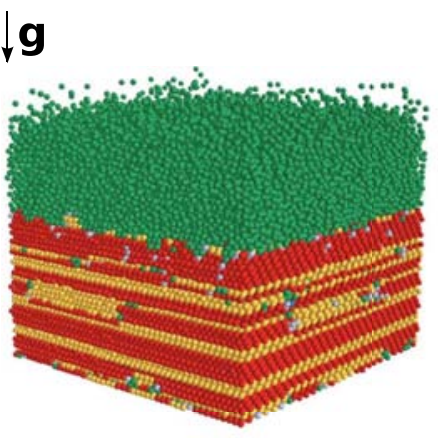

(a) $\mathrm{Pe}=1$

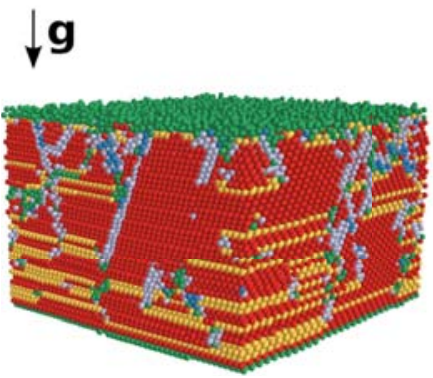

(d) $\mathrm{Pe}=10$

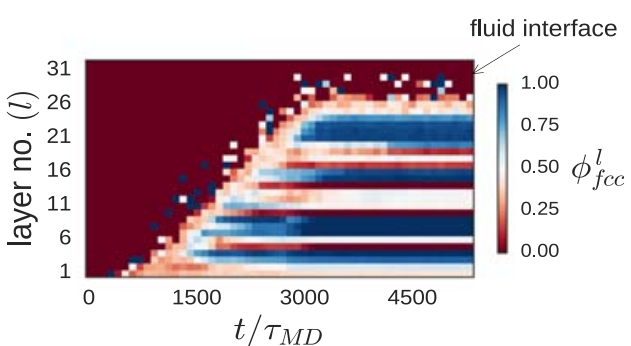

(b) $\mathrm{Pe}=1, \phi_{f c c}^{l}$

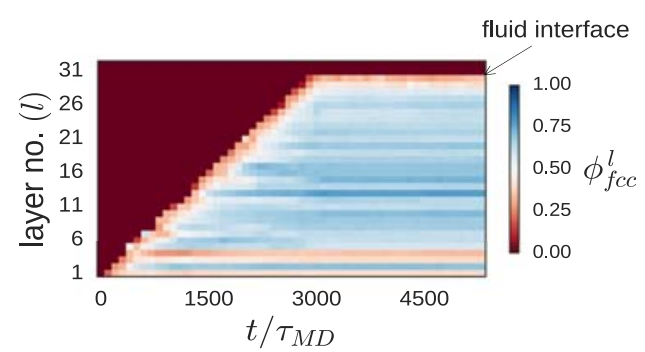

(e) $\mathrm{Pe}=10, \phi_{f c c}^{l}$

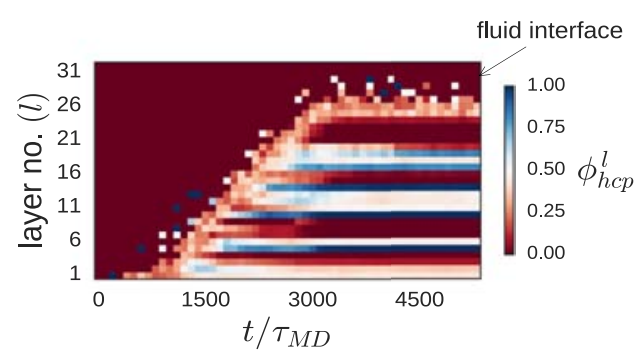

(c) $\mathrm{Pe}=1, \phi_{h c p}^{l}$

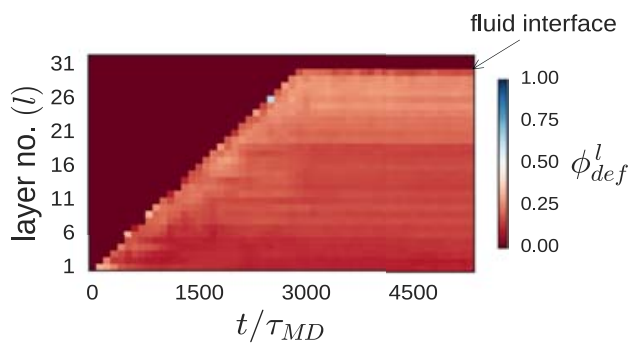

(f) $\mathrm{Pe}=10, \phi_{d e f}^{l}$

FIG. 6. ((a) and (d)) Side view of the final configurations at $t / \tau_{M D}=5300$ of hard spheres sedimenting on an fcc (111) template with lattice spacing $a=1.004 \sigma$ for $\mathrm{Pe}=1$ and 10 as labeled. The color code is the same as that in Fig. 2. ((b) and (e)) Fraction of fcc particles $\phi_{f c c}^{l}$ represented by a colorbar showing the fcc-crystallization of each layer with time. (c) Fraction of hcp particles $\phi_{h c p}^{l}$ represented by a colorbar showing the hcp-crystallization of each layer with time. (f) Fraction of particles belonging to a fault/defect $\phi_{d e f}^{l}$ as indicated by a colorbar, for Pe $=10$, plotted for each layer over time. The metrics of (b) and (c) are intentionally chosen differently from (e) and (f) to demonstrate random stacking of hexagonal layers at low Pe. The dark red region to the left of the four plots discussed above represents the absence of crystalline particles in the layer, i.e., a fluid layer. The initial packing fraction $\eta_{i}=0.02$. 


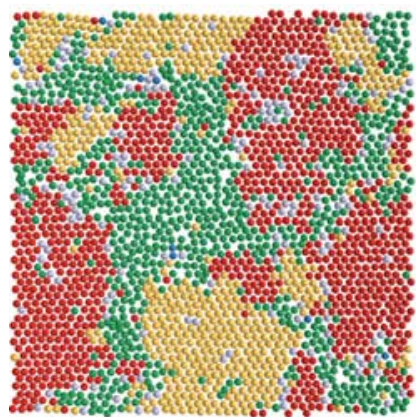

(a) $t / \tau_{M D}=1300$

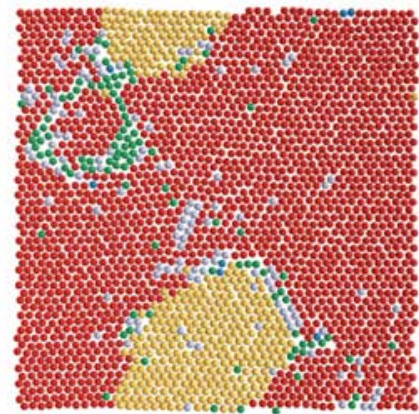

(b) $t / \tau_{M D}=1500$

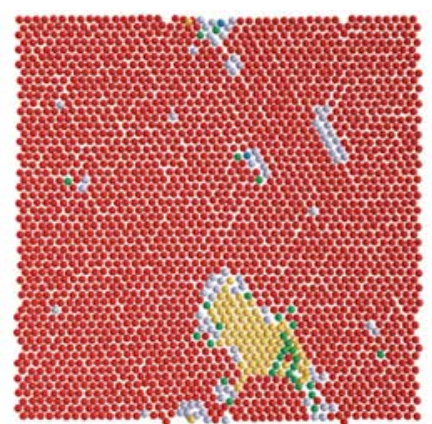

(c) $t / \tau_{M D}=2100$

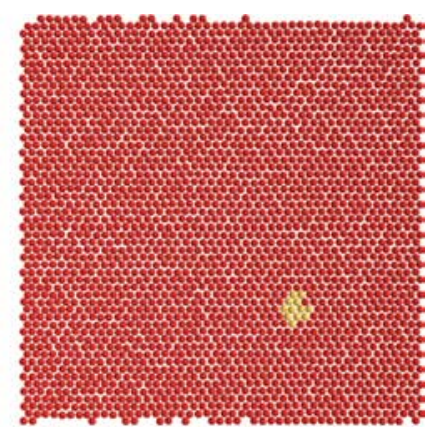

(d) $t / \tau_{M D}=5000$

FIG. 7. Typical configurations of the top view of the fourth layer of the crystalline sediment for different times as labeled for hard spheres sedimenting on a close-packed fcc (111) template at $\mathrm{Pe}=1$. The color code is the same as in Fig. 2.

hexagonal layer, ${ }^{39}$ resulting in a line defect. Hilhorst et al. ${ }^{20}$ argued that the persistence of these line defects across layers and the stacking of subsequent layers of particles lead to two fcc-stacked crystallites which are displaced from each other by a hcp-stacked layer of particles and result in a planar defect. When such a planar defect shifts perpendicular to the defectplane so that the gap on either side of the defect closes, a slanted stacking fault forms. A more detailed description of these defects can be found in the works of Hilhorst $e t$ al. and Marechal et al. ${ }^{21}$ The nature of the sediment is still primarily fcc-like (Fig. 6(e)). We do not observe the formation of the rhcp structure in this case, and we therefore plot $\phi_{\text {def }}^{l}$ in Fig. 6(f) instead of $\phi_{h c p}^{l}$ as we did for $\mathrm{Pe}=1$. The increased number of slanted stacking faults is apparent from the lighter red shade of Fig. 6(f). In the limit of high Pe, the fcc (100) template clearly gives a much better crystal than the fcc (111) (to compare Figs. 2(e) and 6(e)).

Next we move on to the flat wall case. We do not show any of our results for sedimentation on flat walls and only discuss a few observations we made by comparing it to the sedimentation on the fcc (111) template presented above. The interfacial tension between a featureless smooth wall and the fcc (111) hexagonal plane is the lowest among all fcc orientations. ${ }^{40}$ Therefore, when hard spheres sediment on a flat wall, the layer formed at the wall is of hexagonal symmetry. We observe from our simulation snapshots, taken at time intervals of $t / \tau_{M D}=100$, that the first two layers crystallize simultaneously, and once they form, the ensuing crystallization which occurs at the growth front is layer-wise..$^{17,38,41}$ This is then in principle equivalent to the sedimentation on the fcc (111) template and therefore it is interesting to observe how these two cases compare in the low Pe regime. Our simulations of the sedimentation on the flat wall showed a random stacking of hexagonal layers, as observed with the fcc (111) template, at $\mathrm{Pe}=0.5$ with a higher fraction of fcc particles. At $\mathrm{Pe}=1$ we did not observe a random stacking but a primarily fcc-like sediment, in agreement with Marechal et al. In addition to Ref. 21 where only a low Pe was investigated, we looked at a range of Pe for the flat wall case and observed that the fraction of fcc particles $\phi_{f c c}$ steadily decreases with increasing Pe from $\phi_{f c c} \simeq 0.7$ at $\mathrm{Pe}=1$ to $\simeq 0.5$ at $\mathrm{Pe}=10$. At higher Pe for the flat wall case, we see disorder setting in from the bottom layers and propagating to the top.
Interestingly for high Pe, unlike the flat wall, the fcc (111) template appears to restrict the onset of a crystalline to disordered transition in the bottom layers as a result of which the fraction of the orientationally aligned fcc remains largely constant at $\phi_{f c c} \simeq 0.7$ for the entire range of $\mathrm{Pe}$ investigated.

We round off the template comparison with a look at sedimentation on the least dense of all the three templates, i.e., the fcc (110) template, for a low and high Peclet number, Pe $=1$ and 10, respectively. The corresponding snapshots of our EDBD simulations are shown in Figs. 8(a) and 8(d). From Fig. 8(b), we observe that at low $\mathrm{Pe}=1$, the crystalline sediment is fcc-like with $\phi_{f c c} \simeq 0.75$. However, a large number of stacking faults are already seen to appear in the sediment, plotted as defects in Fig. 8(c). However, it is important to note here that the particles form the (111)-oriented fcc, which is of a different orientation than that of the (110) template. This is highly surprising as the sedimenting particles seem to completely ignore the (110) template. Van Blaaderen et al..$^{22}$ and Jensen et $a l .{ }^{27}$ both reported that at low Pe, a crystalline sediment can grow on an fcc (110) template, but they did not study the quality or orientation of the crystal. Similar to the rearrangements of smaller domains observed in the sediment on the fcc (111) template as discussed above, certain layers in this crystalline sediment also show the annealing out of smaller hep domains into larger fcc domains as shown in Fig. 9. However the realignment process takes longer, presumably due to the presence of extended slanted stacking faults in the sediment.

When the sedimentation is faster, i.e., at $\mathrm{Pe}=10$, we observe that (i) disorder sets in at the bottom of the sediment because the template is not sufficiently close-packed to constrain the particles to a layer and (ii) the bottom-most layers are composed of both fcc (100) and (111) like particles, with no clear preference for either. The presence of fcc (100) is likely because it has a lower density mismatch with the template in comparison to fcc (111). Jensen et al. ${ }^{27}$ also discussed high speed centrifugation on the fcc (110) template. They reported that for high sedimentation flux (short time for equilibration and diffusion of particles in-plane), the fcc (110) template is not dense enough to sufficiently constrain the incoming particles in-plane therefore resulting in an eventually crystalline to amorphous crossover. From our simulations, we also observe, 


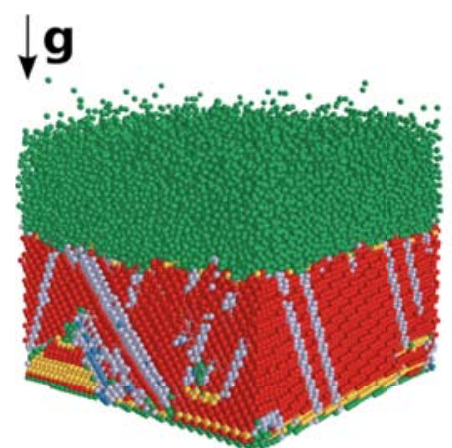

(a) $\mathrm{Pe}=1$

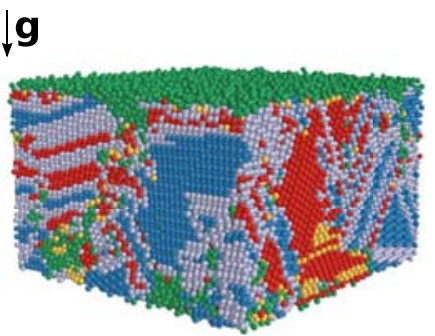

(d) $\mathrm{Pe}=10$

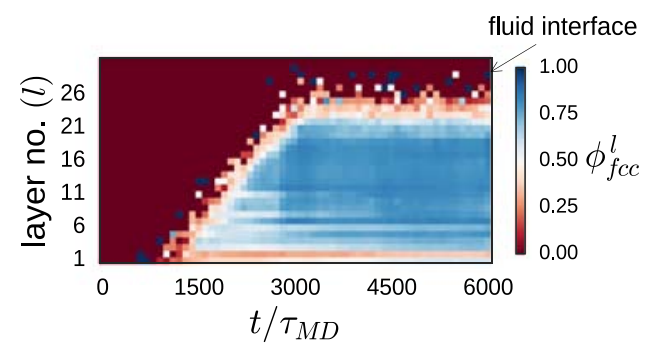

(b) $\mathrm{Pe}=1, \phi_{f c c}^{l}$

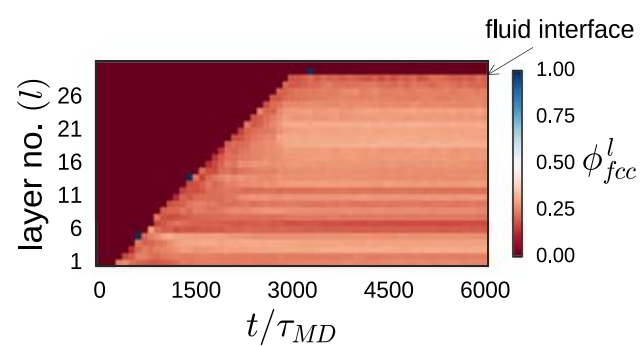

(e) $\mathrm{Pe}=10, \phi_{f c c}^{l}$

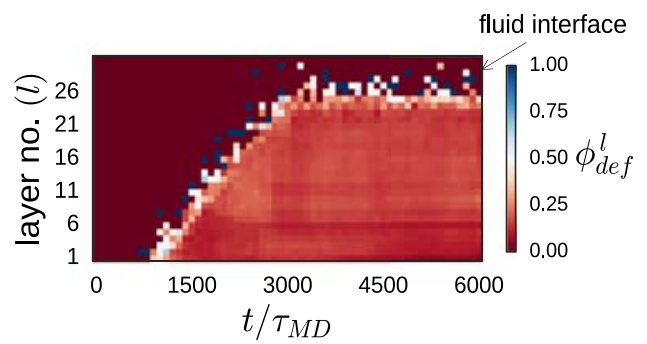

(c) $\mathrm{Pe}=1, \phi_{\text {def }}^{l}$

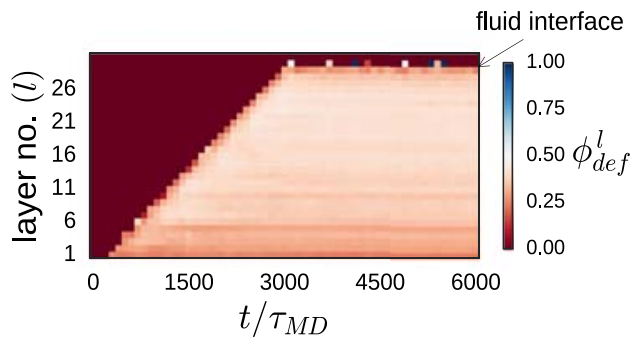

(f) $\mathrm{Pe}=10, \phi_{d e f}^{l}$

FIG. 8. ((a) and (d)) Side view of the final configurations at $t / \tau_{M D}=6000$ of hard spheres sedimenting on an fcc (110) template with lattice spacing $a=1.004 \sigma$ for Pe $=1$ and 10 as labeled. The color code is the same as that in Figs. 2(b) and 2(e). Fraction of fcc particles $\phi_{f c c}^{l}$ represented by a colorbar showing the crystallization of each layer with time. ((c) and (f)) Fraction of particles $\phi_{d e f}^{l}$, again indicated by a colorbar, belonging to a fault/defect plotted for each layer over time. The dark red region to the left of the four plots discussed above represents the absence of crystalline particles in the layer, i.e., a fluid layer. The initial packing fraction $\eta_{i}=0.02$.

commensurate with their findings, a local onset of disorder at high Pe. We observe from the plot shown in Fig. 8(f) that the sediment is highly defective with approximately $50 \%$ defects from the fifth layer upwards. There are distorted crystalline domains making up this defective crystal when there is rapid sedimentation on the fcc (110) template. These domains are not strictly parallel to the template and are colored in lilac in Figure 8(d). We calculated the 3D bond orientational order parameters for these domains and found that they are fcc-like in nature.

To summarize our findings, we estimated the crystallinity of the final sediment for all the cases discussed above by calculating $\phi_{f c c}$, defined as the fraction of similarly oriented fcc particles over the crystalline particles in the whole sediment. A quantitative comparison of the flat wall and the three

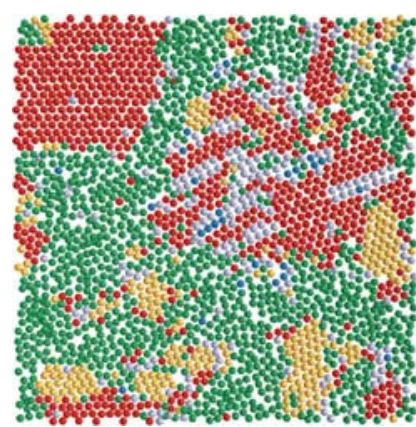

(a) $t / \tau_{M D}=1500$

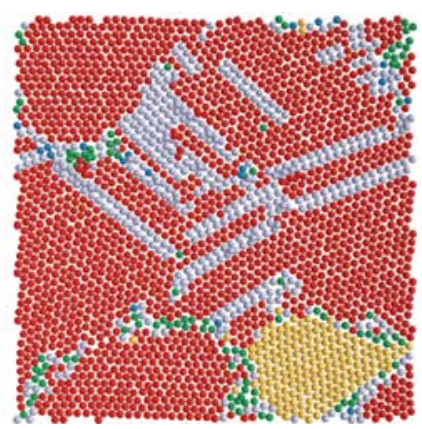

(b) $t / \tau_{M D}=2000$ templates is shown in Fig. 10 from which it is apparent that the best fcc crystals with $\phi_{f c c} \simeq 90 \%$ are found for the fcc (100) template at high Peclet numbers $(\mathrm{Pe}=10)$. This metric has been averaged over three independent simulation runs. The dependence of $\phi_{f c c}$ on Pe is most evident for the fcc (100) template where the percentage of fcc increases with sedimentation rate and the flat wall for which the reverse trend is observed, i.e., the lower the Pe, the better the fcc crystal.

\section{Effect of lattice spacing of the fcc (100) template}

Having established the fcc (100) template as the best template for forming large fcc crystals, we study the effect of

FIG. 9. Typical configurations of the top view of the fourth layer of the crystalline sediment for different times as labeled for hard spheres sedimenting on a close-packed fcc (110) template at $\mathrm{Pe}=1$. The color code is the same as in Fig. 2.

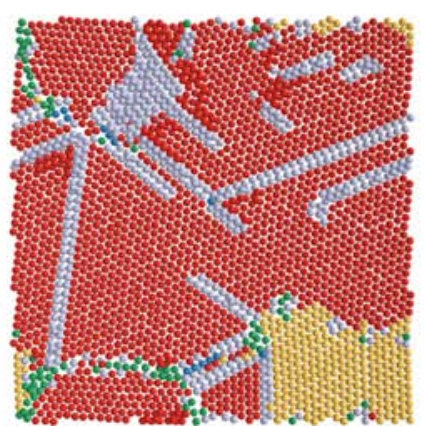

(c) $t / \tau_{M D}=4600$

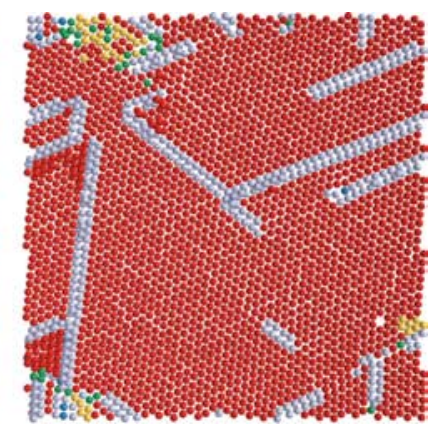

(d) $t / \tau_{M D}=8000$ 


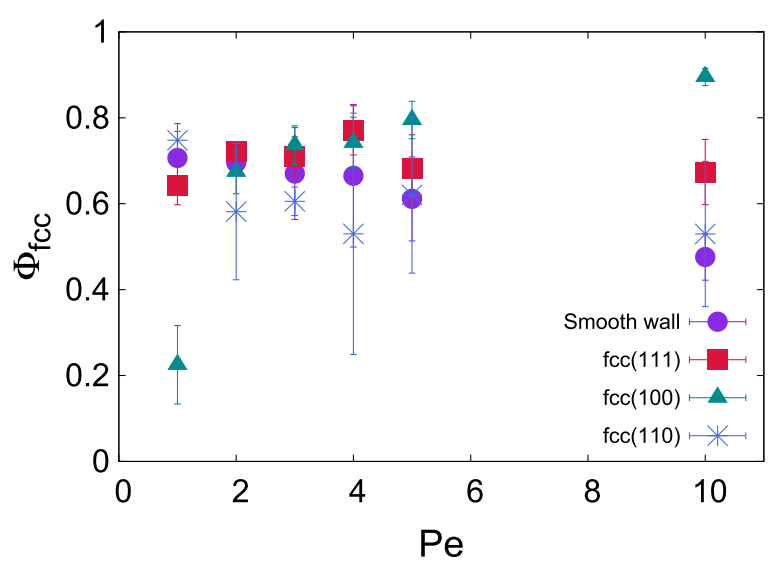

FIG. 10. Fraction of fcc particles $\phi_{f c c}$ in the crystalline sediment as obtained from simulations of sedimenting hard spheres on a smooth wall, an fcc (111), fcc (100), and an fcc (110) template and varying Peclet numbers. The values are averaged over three independent simulations. All simulations are performed for templates with a lattice spacing $a=1.004 \sigma$ and initial packing fraction $\eta_{i}=0.02$.

lattice spacing of this template on the sediment formed. All the results presented in Sections III A and III B correspond to a lattice spacing of $a=1.004 \sigma$. Van Blaaderen et al. ${ }^{22}$ experimented with different lattice spacings of the square template and observed that larger spacings lead to a higher number of defects, thus affecting the quality of the crystal significantly. ${ }^{22}$ Therefore, we simulate sedimentation on a fcc (100) template with a lattice spacing $a=1.1 \sigma$ which corresponds roughly to the density of the bulk crystal at coexistence. The first layer that forms on this template has also square symmetry. The effect of the template is prominent in that the lattice spacing of this layer is also $1.1 \sigma$. Therefore, there is no density mismatch between the template and the crystal formed. The particles in each layer sit on the holes left by the previous layer similar to the close-packed $a=1.004 \sigma$ case. However, there are numerous defects in the layer because of the lower density. This is true for the subsequent layers, which do not rearrange over time. In the case of the close-packed template, the weight of the sediment causes the layer defects to anneal out. In the case of the less dense template $(a=1.1 \sigma)$, this effect is counteracted by the fact that the bridge-site (defect) particles span across the entire cross-sectional area and form a network of closed connected circuits, thus adversely affecting the layer to restructure. Therefore, we see the drop in the overall fraction of fcc particles $\left(\phi_{f c c}\right)$ in the final crystal. In Fig. 11, we plot the fraction of fcc particles $\phi_{f c c}$ as a function of Peclet number for a fcc (100) template with lattice spacing $a=1.004 \sigma$ and $a=1.1 \sigma$. While we find for the template with $a=1.1 \sigma$ that $\phi_{f c c}$ is largest at low Peclet number, i.e., $\mathrm{Pe}=1$, the opposite trend is observed for the close-packed template, which gives the highest $\phi_{f c c}$ for high Peclet number. We explored further the source of lattice mismatch by calculating the pressure at the bottom and the bulk density of an fcc crystal at this pressure. The pressure at the bottom of the sample $(z=0)$ is given by the weight of the sediment per unit area, ${ }^{38}$

$$
\beta P(z=0) \sigma^{3}=g^{*} \rho_{A}^{*}
$$

with $\rho_{A}^{*}=N \sigma^{2} / A$ and $g^{*}=m g \sigma / k_{B} T$. In our simulations, the cross-sectional area of the column is $A \simeq 50 \sigma \times 50 \sigma$ and the

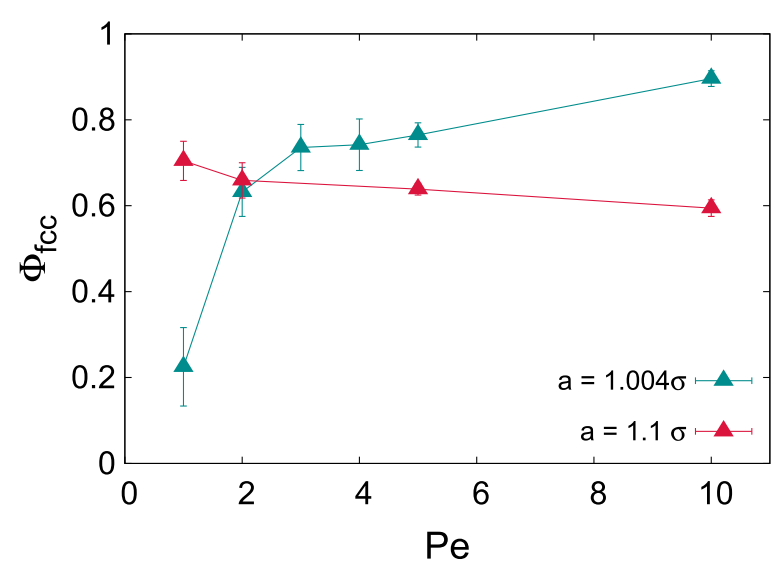

FIG. 11. Fraction of fcc particles $\phi_{f c c}=N_{f c c} / N_{c r}$ in the crystalline sediment of hard spheres settling on a square fcc (100) template with lattice spacing $a=1.004 \sigma$ and $1.1 \sigma$ as a function of Peclet number Pe. The values are averaged over three independent simulations. The lines are guides to the eye. The initial packing fraction $\eta_{i}=0.02$.

number of particles is $N=100000$. With these parameters, $\beta P(z=0) \sigma^{3} \simeq 400$ for $\mathrm{Pe}=10$. We determined the density of the fcc crystal at this pressure using the Speedy equation of state, corresponding to a volume fraction and lattice spacing of 0.732 and $1.0036 \sigma$, respectively. This may explain the superior quality of the fcc crystals formed on the $1.004 \sigma \mathrm{fcc}(100)$ template at $\mathrm{Pe}=10$. Similarly, for $\mathrm{Pe}=1$, we find $\beta P(z=0) \sigma^{3}$ $\simeq 40$. The fcc crystal at this pressure corresponds to a lattice spacing of $\simeq 1.05 \sigma$ (and volume fraction of 0.634 ), which differs significantly from that of the template. This may explain the poor quality of the fcc crystal. It would be interesting to look at several values of the lattice spacing and identify if the optimal value of $a$, which yields the best sediment for a given $\mathrm{Pe}$, is the one with the least mismatch. In the case of the template with the wider lattice spacing, $\phi_{f c c} \simeq 0.70$ remains rather constant as a function of $\mathrm{Pe}$, whereas the close-packed template shows a steady increase of $\phi_{f c c}$ from $\sim 0.5$ at $\mathrm{Pe}=1$ to $\sim 0.9$ for $\mathrm{Pe}=10$. To summarize, the best fcc crystal is obtained by sedimentation on a close-packed square template at $\mathrm{Pe}=10$.

\section{Effect of initial volume fraction}

Finally, we investigate the dependence of the overall fraction of fcc particles $\phi_{f c c}$ on the initial volume fraction $\eta_{i}$ in the case of sedimentation on a close-packed fcc (100) template. Hoogenboom et al. showed from experiments and Marechal et al. ${ }^{21}$ by simulations that the fraction of fcc particles $\phi_{f c c}$ in a sediment deposited on a smooth wall depends strongly on the dimensionless sedimentation flux $\mathrm{J}=\eta_{i} \times \mathrm{Pe}$, which sets the time available for the particles to crystallize at the growth front. The dimensionless sedimentation flux was also used by Jensen et al. ${ }^{27}$ to characterize their sedimentation experiments on different templates and further compare their results on the flat wall with Hoogenboom et al. ${ }^{14}$ and Davis et al. ${ }^{29}$ In Fig. 12, we plot the overall fraction of fcc particles $\left(\phi_{f c c}\right)$ of a crystalline sediment on a square fcc (100) template as a function of Pe for three different initial volume fractions, $\eta_{i}=0.01,0.02$, and 0.104 . We find a strong dependence of $\phi_{f c c}$ on Pe at least for $\eta_{i} \leqslant 0.1$. In the inset of Figure 12, we plot $\phi_{f c c}$ as a function of the dimensionless sedimentation flux $J$, which clearly shows 


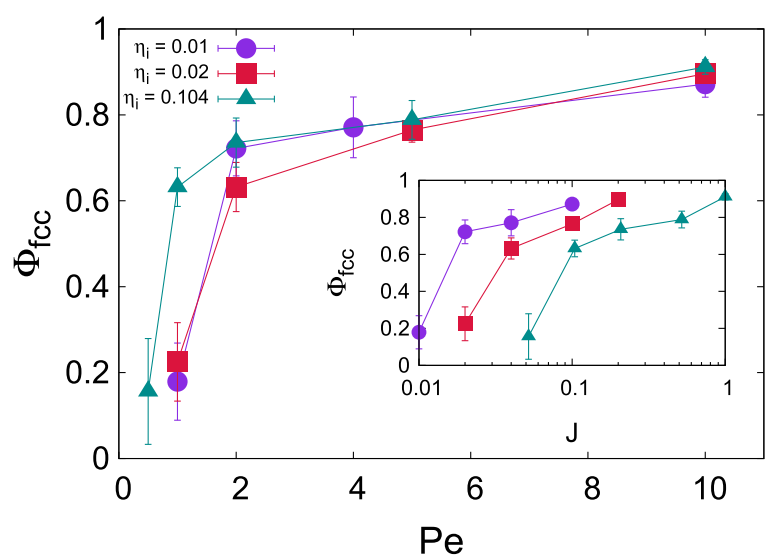

FIG. 12. Fraction of fcc particles $\phi_{f c c}=N_{f c c} / N_{c r}$ in the crystalline sediment of hard spheres settling on the (100) plane of a close-packed fcc crystal as a function of Peclet number Pe for varying initial volume fractions $\eta_{i}$ as labeled. The values are averaged over three independent simulations. The lines are guides to the eye. The inset shows $\phi_{f c c}$ as a function of $J=\eta_{i} \times P e$.

no collapse of the data for varying $\eta_{i}$. This is different from the case of a flat wall as Marechal et al. ${ }^{21}$ observed a collapse of the average stacking parameter for different values of Pe and $\eta_{i}$ but the same value of $J$ (sedimentation flux). In contrast, on the fcc (100) template for Pe $>1$, the fcc crystal quality shows a strong dependence on Pe but not on $\eta_{i}$. For the case $\mathrm{Pe}=1$, we do see an effect of increased packing fraction, indicating that at very slow sedimentation rates, a higher initial packing fraction results in a higher $\phi_{f c c}$.

The reason for the different dependencies lies in the mechanistic differences in the way that the faults in the two crystals rearrange. In the case of the fcc (100) template, the defects are forced to anneal out by the weight of the sediment on top, and this effect is more pronounced at higher Pe. The hard spheres that Marechal et al. studied were sedimented on a smooth wall at low Pe, i.e., the near-equilibrium regime. ${ }^{21}$ In this case, planar defects that shift perpendicular to the plane merge with each other and form slanted stacking faults across layers. Therefore, the quality of the crystal formed is determined by the time available for the layer at the growth front to restructure such that these planar defects can rearrange and not propagate to form $3 \mathrm{D}$ crystal grain boundaries. The time available for this restructuring is set by the value of flux $\mathrm{J}$, and this may explain the collapse of the curves shown in a similar plot to the inset of Fig. 12 for the smooth wall case. ${ }^{21}$ In addition, we observe for the fcc (100) template that for $\mathrm{Pe}=1$, the quality of the crystal improves drastically upon increasing the initial packing fraction such that the stacking faults, as observed in the initial stages of the crystalline sediment, anneal out. In summary, we do not see a strong dependence of the fcc crystal quality on the initial volume fraction for the square fcc (100) template for $\mathrm{Pe} \geq 2$.

\section{CONCLUSIONS}

To summarize, we used event-driven Brownian dynamics simulations to study the crystallization dynamics of hardsphere colloids sedimenting on three different templates, the
(100), (111), and (110) cross-sectional planes of the fcc crystal. We make the following observations.

1. Crystalline sediments grown on the fcc (100) template exhibited the fcc structure with very few defects (see Figs. 2(a) and 2(d)). The fraction of similarly oriented fcc particles $\left(\phi_{f c c}\right)$ increases with settling velocity and is as high as $\simeq 90 \%$ for high settling velocities, Pe $=10$. This surprising observation is in excellent agreement with the experimental work of Jensen $e t$ al. ${ }^{27}$ As noted by the authors, the primary reason for this observation is the absence of stacking degeneracy for the fcc (100) template.

2. While the quality of the crystalline sediment formed on a smooth flat wall is determined by the sedimentation flux, which is a combination of the Peclet number and the initial volume fraction, for the fcc (100) case the Peclet number $(\mathrm{Pe})$ alone determines the quality of the sediment.

3. We also studied the effect of initial volume fraction $\eta_{i}$ for the fcc (100) template with lattice spacing $a=1.004 \sigma$. For $P e>1$, we do not observe a significant effect of the initial packing fraction $\eta_{i}$ on the quality of the sediment. However, at very slow sedimentation rates $(\mathrm{Pe}=1)$, the quality of the crystal improves with the initial packing fraction $\eta_{i}$.

4. When we compare the crystal formed from the sedimentation on two different lattice spacings $(1.004 \sigma$ and $1.1 \sigma)$ of the fcc (100) template, we observe that for lower Pe, a better quality of fcc crystal is formed for the $1.1 \sigma$ template, whereas for higher Pe the close-packed or $1.004 \sigma$ template is clearly superior.

5. We also looked at the fcc (111) template which is the densest one and observed that at low Pe, the layers in the crystalline sediment are randomly "fcc- or hcp-stacked" with only a very few stacking faults. As evidenced by previous simulation and experimental studies, a randomly stacked crystal should eventually rearrange into an fcc crystal over long periods of time,,${ }^{9-11}$ however our simulations are not long enough to make this observation. Sedimentation at high Pe on the fcc (111) template still results in an fcc-like sediment but with several stacking faults.

6. The third template, fcc (110), also gives an fcc-like sediment but with a (111) orientation, which is of a different orientation than that of the (100) template. The template appears to play no role in directing the orientation of the growing crystal. Already at low Pe, several slanted stacking faults are seen in the sediment. Upon increasing the settling velocity $(\mathrm{Pe})$, disorder sets in rapidly.

In summary, we conclude that the best fcc crystals are found in the case of sedimentation on a close-packed fcc (100) template at high Pe, and on an fcc (111) template at low Pe. Our simulations treat the solvent effects via the Brownian noise. The agreement between our simulations and the experiments of Jensen $e t a l .{ }^{27}$ indicates that hydrodynamic interactions do not have a dominant effect on the crystal structures obtained via sedimentation at high Pe on the fcc (100) template. It would also be interesting to look at template assisted sedimentation 
of binary hard-sphere mixtures and we plan to work on these issues in the future.

\section{ACKNOWLEDGMENTS}

This work was done as part of the Industrial Partnership Programme, "Computational Sciences for Energy Research" (Grant No. 13CSER025), of the Foundation for Fundamental Research on Matter (FOM) which is part of the Netherlands Organization for Scientific Research (NWO). This programme is co-financed by Shell Global Solutions International B.V. We thank Wiebke Albrecht, Chris L. Kennedy, and Douglas R. Hayden for a critical reading of the manuscript.

${ }^{1}$ E. C. Nelson, N. L. Dias, K. P. Bassett, S. N. Dunham, V. Verma, M. Miyake, P. Wiltzius, J. A. Rogers, J. J. Coleman, X. Li et al., Nat. Mater. 10, 676 (2011).

${ }^{2}$ A. Van Blaaderen, P. Wiltzius et al., Science 270, 1177 (1995).

${ }^{3}$ P. Schall, I. Cohen, D. A. Weitz, and F. Spaepen, Science 305, 1944 (2004).

${ }^{4}$ P. Schall, I. Cohen, D. A. Weitz, and F. Spaepen, Nature 440, 319 (2006).

${ }^{5}$ L. V. Woodcock, Nature 385, 141-143 (1997).

${ }^{6}$ P. G. Bolhuis, D. Frenkel, S.-C. Mau, and D. A. Huse, Nature 388, 235 (1997).

${ }^{7}$ P. Pusey, W. Van Megen, P. Bartlett, B. Ackerson, J. Rarity, and S. Underwood, Phys. Rev. Lett. 63, 2753 (1989).

${ }^{8}$ L. Filion, M. Hermes, R. Ni, and M. Dijkstra, J. Chem. Phys. 133, 244115 (2010).

${ }^{9}$ S. Pronk and D. Frenkel, J. Chem. Phys. 110, 4589 (1999).

${ }^{10}$ W. K. Kegel and J. K. Dhont, J. Chem. Phys. 112, 3431 (2000).

${ }^{11}$ V. Martelozzo, A. Schofield, W. Poon, and P. Pusey, Phys. Rev. E 66, 021408 (2002).

${ }^{12}$ A. Yethiraj and A. van Blaaderen, Nature 421, 513 (2003).

${ }^{13}$ P. Pusey and W. Van Megen, Nature 320, 340 (1986).

${ }^{14}$ J. P. Hoogenboom, D. Derks, P. Vergeer, and A. van Blaaderen, J. Chem. Phys. 117, 11320 (2002)

${ }^{15}$ J. P. Hoogenboom, P. Vergeer, and A. van Blaaderen, J. Chem. Phys. 119, 3371 (2003).
${ }^{16}$ N. V. Dziomkina and G. J. Vancso, Soft Matter 1, 265 (2005).

${ }^{17}$ I. Ramsteiner, K. E. Jensen, D. A. Weitz, and F. Spaepen, Phys. Rev. E 79, 011403 (2009).

${ }^{18}$ J. Zhu, M. Li, R. Rogers, W. Meyer, R. Ottewill, W. Russel, P. Chaikin et al., Nature 387, 883 (1997).

${ }^{19}$ Z. Cheng, P. Chaikin, J. Zhu, W. Russel, and W. Meyer, Phys. Rev. Lett. 88, 015501 (2001).

${ }^{20}$ J. Hilhorst, J. R. Wolters, and A. V. Petukhov, CrystEngComm 12, 3820 (2010).

${ }^{21}$ M. Marechal, M. Hermes, and M. Dijkstra, J. Chem. Phys. 135, 034510 (2011).

22 A. Van Blaaderen, R. Ruel, and P. Wiltzius, Nature 385, 321 (1997).

${ }^{23}$ M. Heni and H. Löwen, J. Phys.: Condens. Matter 13, 4675 (2001).

${ }^{24}$ Y. Yin, Y. Lu, B. Gates, and Y. Xia, J. Am. Chem. Soc. 123, 8718 (2001).

${ }^{25}$ J. P. Hoogenboom, A. van Langen-Suurling, J. Romijn, and A. van Blaaderen, Phys. Rev. Lett. 90, 138301 (2003).

${ }^{26}$ J. P. Hoogenboom, A. van Langen-Suurling, J. Romijn, and A. van Blaaderen, Phys. Rev. E 69, 051602 (2004).

${ }^{27}$ K. Jensen, D. Pennachio, D. Recht, D. Weitz, and F. Spaepen, Soft Matter 9, 320 (2013).

${ }^{28}$ W.-S. Xu, Z.-Y. Sun, and L.-J. An, J. Chem. Phys. 132, 144506 (2010).

${ }^{29}$ K. Davis, W. Russel, and W. Glantschnig, Science 245, 507 (1989).

${ }^{30}$ P. Strating, Phys. Rev. E 59, 2175 (1999).

${ }^{31}$ A. Scala, T. Voigtmann, and C. De Michele, J. Chem. Phys. 126, 134109 (2007).

${ }^{32}$ R. Piazza, Rep. Prog. Phys. 77, 056602 (2014).

${ }^{33}$ P. R. Ten Wolde, M. J. Ruiz-Montero, and D. Frenkel, Phys. Rev. Lett. 75, 2714 (1995).

${ }^{34}$ M. Heni and H. Löwen, Phys. Rev. Lett. 85, 3668 (2000).

${ }^{35}$ I. Volkov, M. Cieplak, J. Koplik, and J. R. Banavar, Phys. Rev. E 66, 061401 (2002).

${ }^{36}$ S. Auer and D. Frenkel, Phys. Rev. Lett. 91, 015703 (2003).

${ }^{37}$ S. Dorosz and T. Schilling, J. Chem. Phys. 136, 044702 (2012).

${ }^{38}$ T. Biben, R. Ohnesorge, and H. Löwen, Europhys. Lett. 28, 665 (1994).

${ }^{39}$ J.-M. Meijer, V. W. de Villeneuve, and A. V. Petukhov, Langmuir 23, 3554 (2007).

${ }^{40}$ M. Heni and H. Löwen, Phys. Rev. E 60, 7057 (1999).

${ }^{41}$ M. Marechal and M. Dijkstra, Phys. Rev. E 75, 061404 (2007). 